\title{
Reinforcement Efficiency of Cellulose Microfibers for the Tensile Stiffness and Strength of Rigid Low-Density Polyurethane Foams
}

\author{
Jānis Andersons ${ }^{1,2, *}$, Mikelis Kirpluks ${ }^{1}$ (I) and Ugis Cabulis ${ }^{1}$ \\ 1 Polymer Laboratory, Latvian State Institute of Wood Chemistry, 27 Dzerbenes St., LV-1006 Riga, Latvia; \\ mkirpluks@gmail.com (M.K.); cabulis@edi.lv (U.C.) \\ 2 Institute for Mechanics of Materials, University of Latvia, 3 Jelgavas St., LV-1004 Riga, Latvia \\ * Correspondence: janis.andersons@pmi.lv
}

Received: 28 April 2020; Accepted: 10 June 2020; Published: 15 June 2020

check for updates

\begin{abstract}
Rigid low-density closed-cell polyurethane (PU) foams are widely used in both thermal insulation and structural applications. The sustainability of PU foam production can be increased by using bio-based components and fillers that ensure both enhanced mechanical properties and higher renewable material content. Such bio-based foams were produced using polyols derived from rapeseed oil and microcrystalline cellulose (MCC) fibers as filler. The effect of MCC fiber loading of up to $10 \mathrm{wt} \%$ on the morphology, tensile stiffness, and strength of foams has been evaluated. For estimation of the mechanical reinforcement efficiency of foams, a model allowing for the partial alignment of filler fibers in foam struts was developed and validated against test results. It is shown that although applying MCC fibers leads to modest gains in the mechanical properties of PU foams compared with cellulose nanocrystal reinforcement, it may provide a higher content of renewable material in the foams.
\end{abstract}

Keywords: polymer matrix composites; rigid polyurethane foams; microcrystalline cellulose fibers; tensile strength; Young's modulus

\section{Introduction}

Rigid polyurethane (PU) and polyisocyanurate (PIR) foams have found diverse applications in construction, transport, and appliance industries, where their remarkable functional and structural properties are exploited. With sustainability becoming increasingly important, PU production from bio-based feedstock is being pursued in order to increase the renewable material fraction in foams, and also various fillers are applied to achieve the necessary functional characteristics at a lower density of reinforced foams, thus consuming less of the PU polymer [1,2].

The strength and stiffness of PU foams are determined by foam morphology and the mechanical properties of the PU polymer. The latter, depending on the chemical composition, may exhibit great variation in mechanical response, ranging from a flexible to a rigid material. Several factors affect the properties of PU, such as the cross-link density of the polymer matrix, hard/soft segment ratio, isocyanate type, isocyanate index, aromaticity, the presence of dangling side chains, packing and the segmental motion of PU chains [3]. Apart from functionality of the polyol and the type of isocyanate, PU foam properties also depend on such components of foam formulation as blowing agents, surfactants, and catalysts [1]. A convenient way to increase the stiffness of PU is by increasing the cross-link density of polymer via selecting polyols/isocyanates with a higher functional group content per molecule. However, although higher cross-link density results in a larger Young's modulus of the rigid PU foams, it also leads to a reduction in the tensile strength and elongation at break of 
foams [4]. The intermolecular interactions and higher packing of polymer chains usually improve the mechanical properties of PU elastomers and flexible PU foams, whereas the high degree of cross-linking in rigid PU foams hampers the intermolecular conformation of polymer chains. Furthermore, both the strength and stiffness of rigid PU foams can be increased by filling the polymer with micro/nano-size particles, such as nanoclays and carbon nanotubes [5,6], glass fibers [7,8], and carbon fibers [9].

Nano- and microcrystalline cellulose (MCC) fibers can provide efficient mechanical reinforcement not only in monolithic polymer composites, but also in polymer foams due to the advantageous mechanical properties of fibers [10], which are small enough (see e.g., $[10,11]$ ) to be incorporated into cell walls and struts without affecting adversely the foam morphology. An additional benefit of using cellulose reinforcement in biopolymers is an opportunity of increasing the bio-based content of composite materials [11,12]. Cellulose nanofibers possess higher aspect ratio, stiffness, and strength than fibers of microcrystalline cellulose [10], which ensures a greater increase of the mechanical properties of nanofiber-reinforced composite at the same volume fraction of well-dispersed fibers. However, the production of cellulose nanofibers is still associated with high energy consumption and global warming potential [13] (although not exceeding those of other carbon nanomaterials such as carbon nanotubes and graphene [14]), which motivates further studies in the reinforcement efficiency of cellulose microfibers. MCC fibers have been applied as fillers of rigid PU foams comprising soybean [15] and rapeseed oil-based polyols [16,17], and the mechanical response of foams in compression has been studied. A consistent increase of the compressive stiffness and strength of foams with fiber weight fraction varying up to $9 \mathrm{wt} \%$ was found [17], despite the low aspect ratio of MCC fibers.

Relatively little modeling activity has accompanied experimental studies of the mechanical response for micro- and nanocellulose-filled foams, apparently due to complex interaction of cellulose and PU polymer in foams. Along with the aspect ratio and adhesion, the mechanical reinforcement efficiency of anisometric filler particles is strongly affected by their orientation. Cell growth during the foaming process is known to significantly affect the filler alignment in foams. Specifically, biaxial stretching of the cell wall material during cell expansion causes the orientation of fibrous and plate-like fillers along the cell wall, as observed for carbon nanofibers [18] and clay nanoplatelets [19-21]. Such an effect is also predicted by models of fiber motion in the vicinity of growing cells [22,23]. For low-density PU foams, most of the polymer is concentrated in cell struts, and the anisometric fillers would be expected to align with the strut axis due to the stretching of the struts during foam expansion. Indications for such a preferable alignment can be discerned in PU foams filled by, e.g., short milled carbon fibers [9], MCC fibers [15,17], and nanocellulose [24]. Furthermore, the predominant alignment of cellulose nanocrystals parallel to the PU foam rise direction has been reported [25]. Models have been proposed for numerical simulation [26] and analytical prediction [27] of the filler orientation distribution due to stretching of the polymer during the foaming process, demonstrating the additional reinforcing effect imparted by filler alignment.

In the present work, the effect of MCC fiber filler on the tensile strength and stiffness of rigid low-density bio-based PU foams is studied experimentally, thus complementing studies of the response of composite foams in compression [15-17]. The reinforcement efficiency of MCC fibers is evaluated and compared with the literature data for reinforcement of rigid PU foams by cellulose nanofibers. Analytical models for the reinforcement efficiency of foam stiffness and strength reflecting the filler alignment in foam struts are developed and validated.

\section{Materials and Methods}

\subsection{Materials}

For ease of reference and completeness, formulation of the neat bio-based foams [6] is briefly recapitulated below. The polyol system of foams incorporated both polyols derived from rapeseed oil (RO) by amidization with diethanolamine employing zinc acetate as a catalyst (70 pbw) and higher functional polyether polyols based on sorbitol Lupranol 3422 (30 pbw) purchased from BASF 
(Ludwigshafen, Germany). NIAX Silicone L6915LV surfactant (1.5 pbw) produced by Momentive Performance Materials (Leverkusen, Germany). tris-chloropropyl phosphate flame retardant (30 pbw) supplied by Albemarle (Charlotte, NC, USA), and Polycat 5 catalyst ( $1 \mathrm{pbw})$ from Air Products (Halfweg, Netherlands) were also added to the polyol system. As a blowing agent, a mix of water $(1 \mathrm{pbw})$ and cyclo-pentane (12 pbw) was used. Polymeric diphenylmethane diisocyanate IsoPMDI 92140 (164 pbw) supplied by BASF (Ludwigshafen, Germany) was the isocyanate component.

Ultrafine cellulose fibers ARBOCEL UFC 100 from Rettenmaier (Rosenberg, Germany) with a density of $1200 \mathrm{~kg} / \mathrm{m}^{3}$, average fiber length of $8 \mu \mathrm{m}$, and average diameter of $2 \mu \mathrm{m}$ were applied as fillers.

\subsection{Foam Production}

The fillers were added in predetermined quantities $(0 \%, 1 \%, 3 \%, 5 \%, 7 \%$, and $10 \%$ of neat foam polymer weight) to the blend of polyols, surfactant, catalyst, and flame retardant and stirred by mechanical mixer at $200 \mathrm{rpm}$ for $3 \mathrm{~min}$. Subsequently, the blowing agent was added, thus completing the polyol system. The foams were produced by adding isocyanate component, mixing for 10 to $15 \mathrm{~s}$, and pouring the mixture into plastic molds of dimensions $20 \times 30 \times 10 \mathrm{~cm}$ for free foaming. The polymerization reaction took place at room temperature and was completed within about 3 to $5 \mathrm{~min}$.

\subsection{Foam Characterization}

Upon setting of the foams, foam blocks were removed from the molds and conditioned at room temperature for $24 \mathrm{~h}$. Since a denser surface layer had formed in the blocks produced, it was cut off before making specimens for tests.

Foam morphology was characterized by scanning electron microscopy (SEM). Foam samples of dimensions $1 \times 1 \times 0.2 \mathrm{~cm}$ were cut and sputtered with gold by using an Emitech K550X sputter coater (Emitech Ltd, Ashford, UK). Images of the surface of foam slices parallel to the foam rise direction were taken using a Tescan TS 5136 MM (TESCAN ORSAY HOLDING, a.s., Brno, Czech Republic, SEM) with a secondary electron detector; accelerating voltage was set to $15 \mathrm{kV}$, and the working distance was $15 \mathrm{~mm}$. Representative micrographs of foams are presented in Figure 1.

The images obtained were processed with a Vega TC software (version 2.9.9.21, TESCAN R\&D Software Group, Brno, Czech Republic), and cell dimensions in the foam rise (cell length) and transverse (cell width) directions were measured. The average values and standard deviations of cell dimensions, as well as cell length and width ratio $R$ characterizing geometrical anisotropy of foam cells, are listed in Table 1.

Table 1. Geometrical characteristics of foam cells as a function of microcrystalline cellulose fiber loading.

\begin{tabular}{cccc}
\hline Fiber Loading, wt \% & Cell Length, $\boldsymbol{\mu m}$ & Cell Width, $\boldsymbol{\mu m}$ & Shape Anisotropy $\boldsymbol{R}$ \\
\hline 0 & $745(130)^{1}$ & $480(73)$ & $1.56(0.18)$ \\
1 & $567(104)$ & $364(55)$ & $1.56(0.17)$ \\
3 & $514(166)$ & $345(90)$ & $1.48(0.18)$ \\
5 & $565(130)$ & $362(65)$ & $1.56(0.18)$ \\
7 & $502(105)$ & $333(56)$ & $1.51(0.17)$ \\
10 & $498(149)$ & $337(82)$ & $1.47(0.16)$ \\
\hline
\end{tabular}

${ }^{1}$ Standard deviation is given in parentheses.

For mechanical tests, foam blocks were cut into slices along the foam rise direction, and the apparent density of each of the foam slices was determined. Specimens of dog-bone shape with rectangular test sections of $85 \mathrm{~mm}$ length, $22 \mathrm{~mm}$ width, and $20 \mathrm{~mm}$ thickness were machined from the foam slices for tests in the direction transverse to the foam rise.

Tensile tests were performed in stroke control at a loading rate corresponding to $10 \% / \mathrm{min}$ in the gauge section by a servo-hydraulic test machine with a $1 \mathrm{kN}$ load cell. The strain in the loading 
direction was measured by an extensometer MTS 634.25F-24 (MTS Systems Corporation, Eden Prairie, MN, USA) with a $50 \mathrm{~mm}$ gauge length.

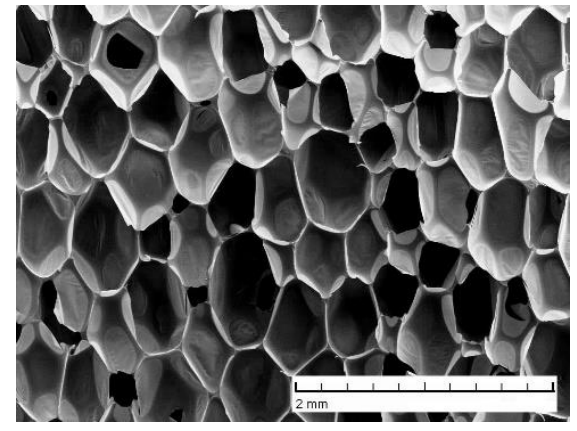

(a)

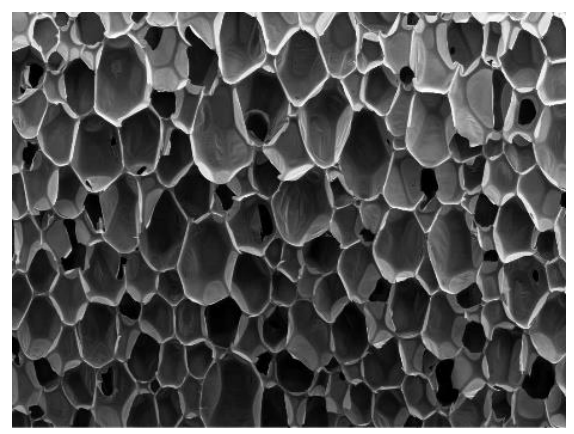

(c)

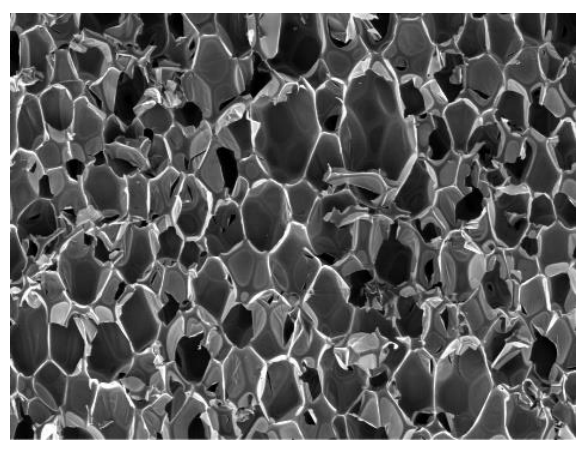

(e)

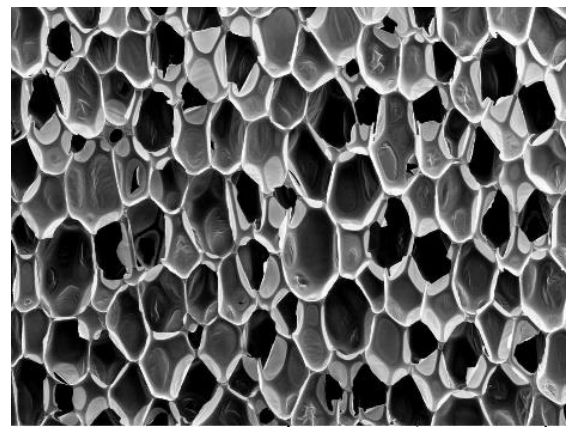

(b)

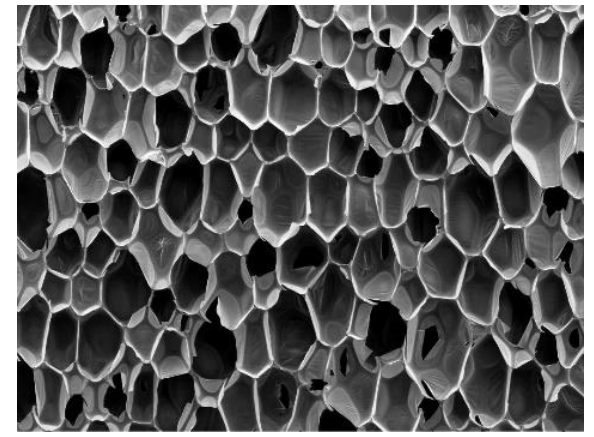

(d)

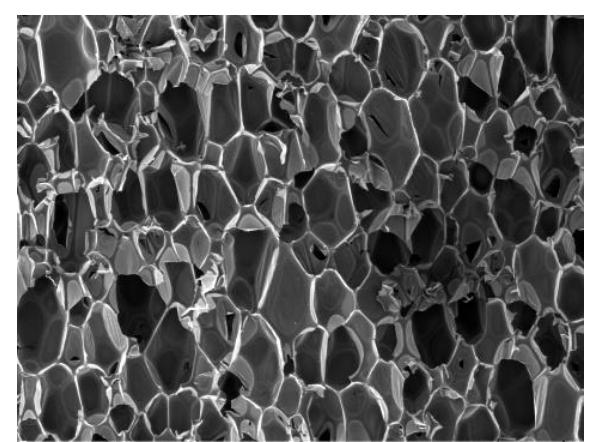

(f)

Figure 1. SEM images of foam cross-section in a plane aligned with the foam rise direction (the vertical direction in the pictures) at MCC fiber filler content of: (a) $0 \mathrm{wt} \%$ (neat foams); (b) $1 \mathrm{wt} \%$; (c) $3 \mathrm{wt} \%$; (d) $5 \mathrm{wt} \%$; (e) $7 \mathrm{wt} \%$; (f) $10 \mathrm{wt} \%$.

\section{Model}

\subsection{Mechanical Reinforcement Efficiency of Foams}

The mechanical properties of polymer foams are determined by the foam porosity, anisotropy of foam cells, and the mechanical properties of the monolithic solid material forming cell walls and struts [28]. The introduction of reinforcing particles is primarily aimed at modifying foam characteristics via improving the properties of the solid polymer. However, the presence of filler can also affect the porosity and morphology of foams, e.g., due to the altering of viscosity of the foaming polymer or by filler particles acting as the bubble nucleation centers. Such changes in composite foam density and morphology also affect the mechanical properties of foams to an extent that can be commeasurable with mechanical reinforcement [29]. To separate the mechanical reinforcement effect from that of 
foam density variation, coefficients of modulus and strength enhancement defined as the ratio of the respective characteristics of composite and neat foams at the same apparent foam density have been introduced [30]. Furthermore, the degree of mechanical reinforcement for foam stiffness has been defined [31] so that the variation in cell shape (characterized by the shape anisotropy $R$ ) between neat and composite foams is also taken into account.

Mechanical reinforcement efficiency for stiffness $\Gamma_{E}$ is defined as the ratio of composite foam modulus $E_{c f}$ and neat foam modulus $E_{f}$, the latter corresponding to the density $\rho_{c f}$ and geometrical anisotropy $R_{c f}$ of composite foams [31]:

$$
\Gamma_{E}=\frac{E_{c f}}{E_{f}\left(\rho_{c f}, R_{c f}\right)} .
$$

The neat foam stiffness can be expressed by a power function of foam density $\rho_{f}[28,31]$,

$$
E_{f}=c_{E} E_{S}\left(\frac{\rho_{f}}{\rho_{s}}\right)^{n_{E}} f_{E}(R)
$$

where $E_{S}$ and $\rho_{s}$ designate the stiffness and density of the solid monolithic foam strut and wall material, $f_{E}(R)$ is a function reflecting the effect of cell shape anisotropy on foam stiffness, and $c_{E}, n_{E}$ are constants to be determined via modeling [28] or by approximating the experimental data by Equation (2) [31]. Assuming that the dependence of stiffness of composite foams on their apparent density and geometrical anisotropy can be described by the same relation, Equation (2), upon substitution of neat solid material characteristics by the modulus $E_{\mathcal{~ S S}}$ and density $\rho_{\mathcal{~ S S}}$ of solid composite cell strut material:

$$
E_{c f}=c_{E} E_{c s}\left(\frac{\rho_{c f}}{\rho_{c s}}\right)^{n_{E}} f_{E}\left(R_{c f}\right)
$$

and inserting foam stiffness expressions Equations (2) and (3) into Equation (1), the following relation for $\Gamma_{E}$ is obtained:

$$
\Gamma_{E}=\frac{E_{c s}}{E_{s}}\left(\frac{\rho_{s}}{\rho_{c s}}\right)^{n_{E}}
$$

Further, expressing neat and composite monolithic material moduli $E_{S}$ and $E_{C S}$ from Equations (2) and (3), respectively, and substituting into Equation (4), the mechanical reinforcement efficiency for foam stiffness is derived in terms of foam characteristics as follows:

$$
\Gamma_{E}=\frac{E_{c f}}{E_{f}}\left(\frac{\rho_{f}}{\rho_{c f}}\right)^{n_{E}} \frac{f_{E}(R)}{f_{E}\left(R_{c f}\right)} .
$$

Similarly, the mechanical reinforcement efficiency factor for foam strength $\Gamma_{\sigma}$ can be defined as the ratio of composite, $\sigma_{c f}$, and neat foam strength, $\sigma_{f}$, with neat foams having the same density and geometric anisotropy as the composite foams:

$$
\Gamma_{\sigma}=\frac{\sigma_{c f}}{\sigma_{f}\left(\rho_{c f}, R_{c f}\right)} .
$$

For low-density foams exhibiting brittle fracture or failing by the appearance of plastic hinges in the struts, foam strength is proportional to strength $\sigma_{s}$ of the solid strut material [28]. The foam strength can be expressed as

$$
\sigma_{f}=c_{\sigma} \sigma_{s}\left(\frac{\rho_{f}}{\rho_{s}}\right)^{n_{\sigma}} f_{\sigma}(R)
$$


where $c_{\sigma}, n_{\sigma}$ are foam morphology-related constants, and function $f_{\sigma}(R)$ allows for geometrical anisotropy effect on foam strength [28]. Assuming as above that relation Equation (7), upon replacing the relevant neat polymer characteristics by those of the composite solid, holds also for composite foam strength, it follows that the strength reinforcement efficiency according to Equation (6) is given by

$$
\Gamma_{\sigma}=\frac{\sigma_{c s}}{\sigma_{s}}\left(\frac{\rho_{s}}{\rho_{c s}}\right)^{n_{\sigma}}
$$

and alternatively, via foam properties:

$$
\Gamma_{\sigma}=\frac{\sigma_{c f}}{\sigma_{f}}\left(\frac{\rho_{f}}{\rho_{c f}}\right)^{n_{\sigma}} \frac{f_{\sigma}(R)}{f_{\sigma}\left(R_{c f}\right)}
$$

The reinforcement efficiency factors Equations (5) and (9) enable the separation of the purely mechanical effect of reinforcing particles on foam stiffness and strength from that caused by alteration in foam density and morphology, whereas Equations (4) and (8) provide a link between foam reinforcement efficiency and the properties of the solid composite material forming cell struts.

\subsection{Stiffness and Strength of Foam Struts}

In low-density closed-cell PU foams, $80 \%$ to $96 \%$ of the polymer is concentrated in cell struts [32,33]; therefore, the effect of cell walls on the mechanical properties can be neglected for a close conservative estimate of foam stiffness and strength [34]. The mechanical characteristics of such foams are determined by the axial stiffness and strength of foam struts via Equations (2) and (7) [28]. Hence, the mechanical reinforcement efficiency of foams can be predicted by Equations (4) and (8) once the composite strut properties are known. The latter can be evaluated by elementary micromechanical models as described below.

\subsubsection{Young's Modulus}

Young's modulus $E_{c s}$ of a fiber-reinforced composite strut can be related to the Young's modulus of polymer matrix $E_{s}$, axial modulus of the reinforcing fibers $E_{A}$, and fiber volume fraction $v_{f}$ by a rule of mixtures type of relationship:

$$
E_{c s}=\eta_{o E} \eta_{l E} E_{A} v_{f}+\left(1-v_{f}\right) E_{s}
$$

where $\eta_{o E}$ designates the fiber orientation factor and $\eta_{I E}$ is the fiber length efficiency factor. The latter can be expressed by a shear-lag model via the aspect ratio (i.e., length-to-diameter ratio) $\kappa$ of the reinforcing fibers and stress transfer rate between the fiber and matrix $\beta$ as follows:

$$
\eta_{l E}=1-\frac{\tanh (\beta \kappa)}{\beta \kappa} .
$$

A number of analytical expressions for the stress transfer rate of various complexity and accuracy have been derived; however, they are mostly applicable to composites of relatively high fiber volume content. Since filler loading is usually relatively low in polymer foams, we use the relation for $\beta$ shown to be accurate even for vanishing fiber volume fraction [35]

$$
\beta^{2}=\frac{2}{E_{A} E_{s}} \frac{E_{A} v_{f}+E_{s} v_{m}}{\frac{v_{m}}{4 G_{A}^{*}}-\frac{1}{2 G_{s}}\left(\frac{v_{m}}{2}+1+\frac{\ln \left(v_{f}+\chi\right)}{v_{m}}+\frac{v_{m}}{r D}\right)} .
$$

In Equation (12), $v_{m}$. stands for the matrix volume fraction, $v_{m}=1-v_{f}, G_{A}^{*}=G_{A} /\left(1+2 G_{A} / r D\right)$ is the effective fiber shear modulus, $G_{A}$ and $r$ are fiber shear modulus and radius, respectively, $G_{S}$ 
denotes the shear modulus of the matrix, $D$ designates a stiffness parameter of the fiber/matrix interface, and $\chi=0.009$. is a numerically determined constant ensuring the accuracy of the expression for $\beta$ at low fiber volume fractions [35].

If the distribution density $p(\theta)$. of the fiber orientation angle $\theta$, i.e., the angle between fiber and strut axes, is known, fiber orientation factor $\eta_{o E}$. can be evaluated according to Krenchel's approach (see e.g., [36]):

$$
\eta_{o E}=\int_{0}^{\pi / 2} p(\theta) \cos ^{4} \theta \sin \theta d \theta
$$

\subsubsection{Strength}

For the axial tensile strength $\sigma_{c s}$ of a composite foam strut, the Fukuda and Chou model [37] in its modified form [38] is applied, expressing $\sigma_{\mathcal{C S}}$ as a weighted sum of fiber axial strength $\sigma_{A}$ and matrix strength $\sigma_{s}$ :

$$
\sigma_{c s}=\eta_{o \sigma} \eta_{l \sigma} v_{f} \sigma_{A}+\left(1-v_{f}\right) \sigma_{s}
$$

and employing fiber orientation, $\eta_{o \sigma}$, and length, $\eta_{l \sigma}$, efficiency factors for strength. The latter is presented in terms of reinforcing fiber length $l$ and critical length $l_{c}$ as

$$
\eta_{l \sigma}=\left\{\begin{array}{ll}
l / 2 l_{c} & l \leq l_{c} \\
1-l_{c} / 2 l & l>l_{c}
\end{array} .\right.
$$

where

$$
l_{c}=\sigma_{A} r / \tau
$$

and $\tau$ stands for the interfacial shear strength (IFSS). Assuming a vanishingly small width of the critical zone, the fiber orientation factor $\eta_{o \sigma}$ is also given by Equation (13) [38].

\subsubsection{Fiber Orientation Distribution}

Upon vigorous mixing of the PU foam components, the orientations of filling fibers in the mixture are likely to be random, with uniform spatial and orientation distribution. During foaming, bubble nucleation and growth causes stretching of the struts, imparting preferential orientation of the fibers along the strut axis. We assume that the initial orientation distribution of filler is uniform, each strut undergoes isochoric stretching along its axis, and the anisometric filler particles are subjected to an affine rotation due to this stretching [27]. Then, the resulting fiber orientation distribution is symmetric about the strut axis, and the distribution density of the fiber orientation angle $\theta$ is given by (see, e.g., [39]):

$$
p(\theta)=\frac{\lambda^{3}}{\left[\lambda^{3}+\left(1-\lambda^{3}\right) \cos ^{2} \theta\right]^{3 / 2}}
$$

where $\lambda$ is the stretch ratio of a strut, i.e., the degree to which the strut has been stretched during foaming. Fiber orientation factor $\eta_{o}=\eta_{o E}=\eta_{o \sigma}$ is obtained upon the substitution of $p(\theta)$ into Equation (13) and integration:

$$
\eta_{o}=\frac{\lambda^{3}}{2}\left(\frac{1+2 \lambda^{3}}{\left(\lambda^{3}-1\right)^{2}}-\frac{3 \lambda^{3}}{\left(\lambda^{3}-1\right)^{5 / 2}} \sec ^{-1} \lambda^{3 / 2}\right) .
$$

Since stretching of the structural elements of foams results from foam expansion, the stretch ratio should be a function of the foam expansion ratio $V_{\text {foams }} / V_{\text {solid }}$, i.e., the relative change in material volume during foaming; the expansion ratio is the inverse of the relative density of foams $\gamma, V_{\text {foams }} / V_{\text {solid }}=$ $\rho_{s} / \rho_{f}=1 / \gamma$. Assuming that the stretch ratio $\lambda$ is the same for all the foam struts regardless of 
their length and orientation, it can be approximately related to the foam expansion ratio so that $\lambda^{3}=V_{\text {foams }} / V_{\text {solid }}=1 / \gamma$, from which

$$
\lambda=1 / \sqrt[3]{\gamma} .
$$

\section{Results and Discussion}

As can be discerned in Figure 1 and Table 1, the presence of MCC filler caused a reduction in cell size by approximately $30 \%$, which suggests that cellulose fibers acted as a nucleation agent facilitating the formation of bubbles during foaming. By contrast, the shape anisotropy of foam cells remained almost constant, exhibiting a very slight reduction with increasing fiber loading. The renewable material content of neat foams amounted to ca. $20 \mathrm{wt} \%$. Incorporation of the cellulose filler increased the sustainable material fraction in composite foams up to about $28 \mathrm{wt} \%$ at the highest filler loading considered.

The MCC fibers had a positive effect on the tensile strength and stiffness of the foams, as seen in Table 2. Specifically, at $10 \mathrm{wt} \%$ fiber loading, the Young's modulus of composite foams exceeded that of neat ones by about $45 \%$, while the increase of strength amounted to ca. $17 \%$. However, the larger fiber content was accompanied also by a growing apparent density of foams, presumably due to an increase in the viscosity of the polyol premix [17].

Table 2. Foam density, stiffness, and strength as a function of MCC fiber loading.

\begin{tabular}{ccccc}
\hline $\begin{array}{c}\text { Fiber Loading, wt } \\
\mathbf{\%}\end{array}$ & $\begin{array}{c}\text { Foam Density, } \\
\mathbf{~} \mathbf{g} / \mathbf{m}^{\mathbf{3}}\end{array}$ & $\begin{array}{c}\text { Young's Modulus, } \\
\mathbf{M P a}\end{array}$ & $\begin{array}{c}\text { Tensile Strength, } \\
\mathbf{k P a}\end{array}$ & $\begin{array}{c}\text { Strain at Failure, } \\
\mathbf{\%}\end{array}$ \\
\hline 0 & $33.1(1.3)^{1}$ & $3.36(0.76)$ & $127(20)$ & $6.6(0.3)$ \\
1 & $33.0(0.5)$ & $3.10(0.27)$ & $137(12)$ & $7.6(0.6)$ \\
3 & $34.3(0.5)$ & $3.90(0.29)$ & $142(7)$ & $6.4(1.0))$ \\
5 & $33.4(0.5)$ & $3.66(0.25)$ & $134(12)$ & $5.9(0.4)$ \\
7 & $35.6(0.7)$ & $4.74(0.67)$ & $152(13)$ & $5.6(0.7)$ \\
10 & $35.8(0.5)$ & $4.86(0.47)$ & $148(16)$ & $5.0(0.6)$ \\
\hline
\end{tabular}

${ }^{1}$ Standard deviation is given in parentheses.

In order to estimate the mechanical reinforcement efficiency of foams by Equations (5) and (9), exponents $n_{E}, n_{\sigma}$ and shape anisotropy functions $f_{E}, f_{\sigma}$ entering the respective expressions need to be specified. For stiffness and strength in the direction transverse to the foam rise, the latter can be expressed in the form $f_{E}(R)=\left(R+1 / R^{2}\right) /(R+2)^{2}$ and $f_{\sigma}(R)=(R+1)(R /(R+2))^{3 / 2} / R^{2}$ respectively by the rectangular-cell model; see [28,34]. As concerns the exponents of the density dependence of foam characteristics, we evaluated them by fitting Equations (2) and (7) to the experimental data for neat foams of the same formulation reported in [34]. The respective data are shown in Figure 2 together with best-fit approximations corresponding to $n_{E}=1.9$ and $n_{\sigma}=1.2$. Notably, the value of the power-law exponent for the density dependence of foam stiffness is very close to the one derived for low-density open-cell foams and amounting to $n_{E}=2$, while the exponent for strength is lower than the predicted $n_{\sigma}=1.5[28]$.

Mechanical reinforcement efficiency factors, evaluated by Equations (5) and (9) using the parameter values and the experimental data of Table 2, are presented in Figure 3 as functions of MCC fiber loading. It is seen that the correction for foam density and shape anisotropy has revealed the maximum reinforcement efficiency for stiffness of about $20 \%$ at 7 and $10 \mathrm{wt} \%$ fiber loading, the rest of the apparent gain in stiffness reflected in Table 2 being caused primarily by an increase in foam density due to the presence of the filler. The strength reinforcement efficiency, Figure $3 b$, is also markedly smaller than the apparent gain in strength suggested by the data in Table 2 . 


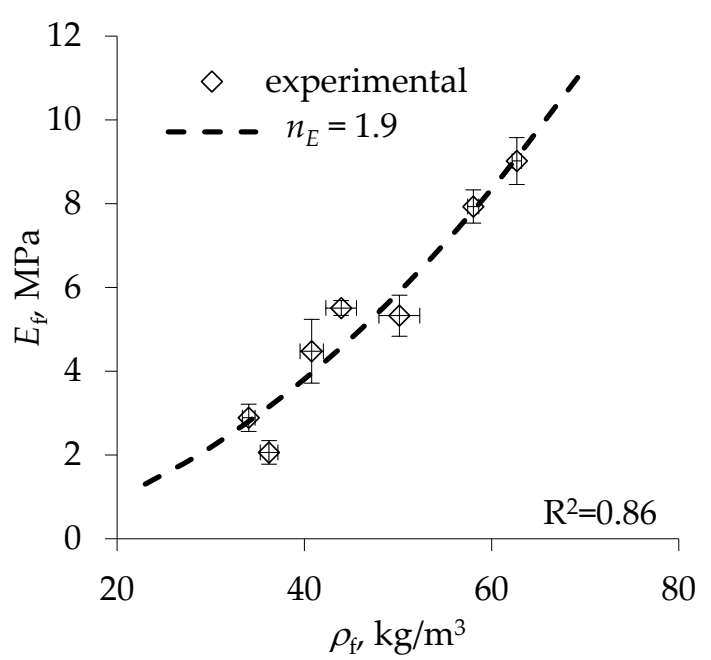

(a)

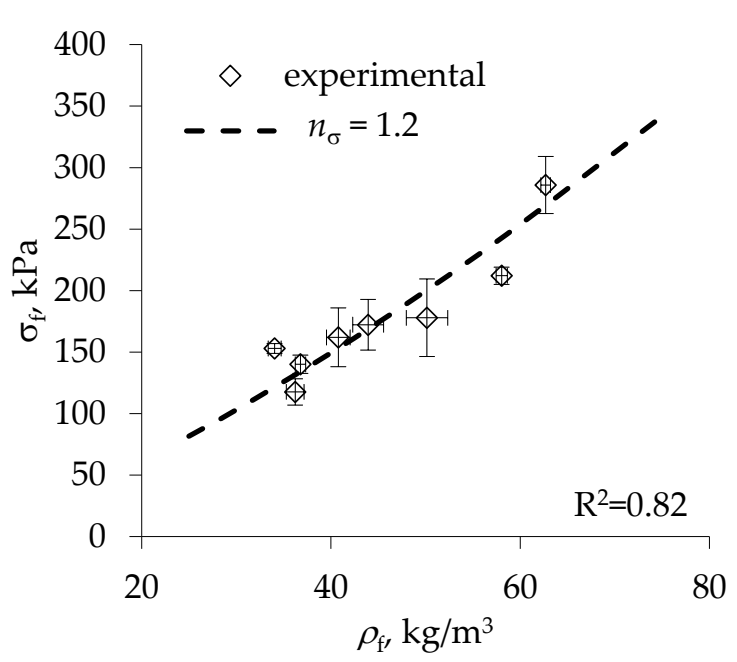

(b)

Figure 2. Variation of neat foam (a) modulus and (b) strength under tension in the transverse direction with foam density [34] and approximations of the data by Equations (2) and (7), as shown by dashed lines.

For theoretical estimation of the mechanical reinforcement efficiency factor of foam stiffness according to Equation (4), we rely on the MCC fiber properties reported in the literature. The axial modulus $E_{A}$. of deagglomerated, rod-like MCC fibers was evaluated at $25 \mathrm{GPa}$ [40], and shear modulus $G_{A}$. of MCC at vanishing porosity was estimated as 3.5 GPa [41]. Due to good adhesion expected between the cellulose and PU polymer, the fiber/matrix interface stiffness parameter $D \rightarrow \infty$. implying perfect interface [35] is used in Equation (12). The stiffness and density of the neat polymer amounted to $E_{s}=2.3 \mathrm{GPa}$ and $\rho_{s}=1210 \mathrm{~kg} / \mathrm{m}^{3}$ [34]. The fiber volume fraction $v_{f}$ in the solid polymer is expressed via fiber weight fraction $c$ (of neat polymer weight) as $v_{f}=c /\left(c+\varrho_{M C C} / \varrho_{s}\right)$ with $\varrho_{M C C}$ denoting the density of MCC fibers. Since the foam expansion ratios, calculated using the foam density values of Table 2, exhibited little variation ranging between 33.5 and 36.4, the average expansion ratio was applied to evaluate the fiber orientation factor by Equations (18) and (19), yielding $\eta_{0}=0.69$. The resulting dependence of $\Gamma_{E}$ on MCC fiber loading according to Equation (4) is plotted in Figure 3a by a solid line. A good agreement of the theoretical prediction with the efficiency factor values derived from test results is seen.

Concerning the reinforcement efficiency for strength, hydrogen [42] or even covalent [43] bonding between the cellulose and PU matrix ensures good adhesion; hence, stress transfer of the fiber/matrix interface in shear is likely to be limited by yielding of the PU polymer. Then, the IFSS $\tau$ entering the expression of fiber length efficiency factor Equation (15) can be roughly approximated by the shear yield strength of the solid polymer [44]. According to the von Mises yield criterion, the yield strength in shear is related to the tensile yield strength $\sigma_{s}$ as $\tau=\sigma_{s} / \sqrt{3}$; for the neat solid polymer, $\sigma_{s}=61.5 \mathrm{MPa}$ [34]. In the absence of experimental data regarding the axial tensile strength of MCC fibers, we substituted wood fiber strength [10] for $\sigma_{A}$ into Equation (16) to estimate the lower-bound value of the critical length of MCC fiber and found that $l_{c}>l$. It then follows from Equations (14) and (15) that the composite strut strength $\sigma_{c s}=\eta_{o \sigma} \kappa \tau v_{f}+\left(1-v_{f}\right) \sigma_{s}$. Using this relation, the predicted mechanical reinforcement efficiency for strength according to Equation (8) is plotted in Figure $3 \mathrm{~b}$ by the solid line. It is seen that $\Gamma_{\sigma}$ derived from foam tests and from composite strength estimates agree well and are both rather low - the predicted reinforcement efficiency factor for $10 \mathrm{wt} \%$ loading amounts to 1.06 , while the experimental $\Gamma_{\sigma}$ values are ca. 1.09 and 1.03 for $7 \mathrm{wt} \%$ and $10 \mathrm{wt} \%$ fiber content, respectively. For comparison, the predicted reinforcement efficiency for $\eta_{o}=1$ is also displayed in Figure 3, showing the level of $\Gamma_{E}$, $\Gamma_{\sigma}$ attainable at a perfect fiber alignment with the strut axis. 


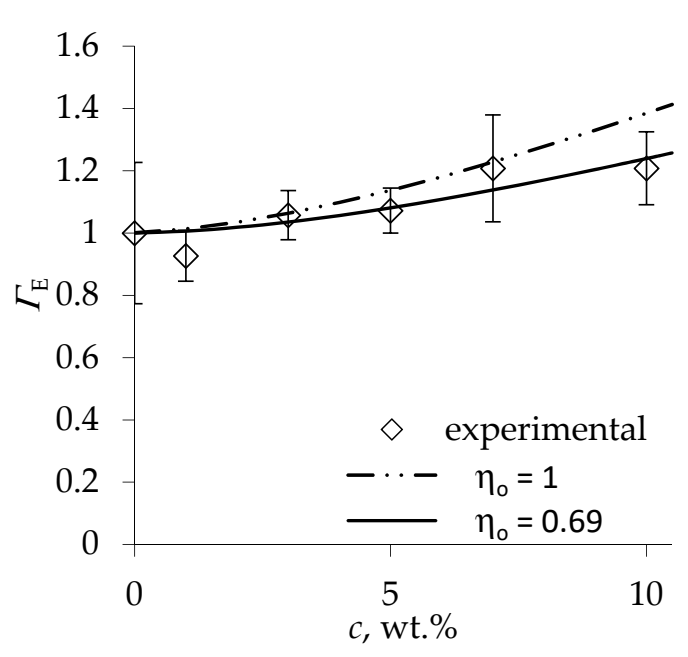

(a)

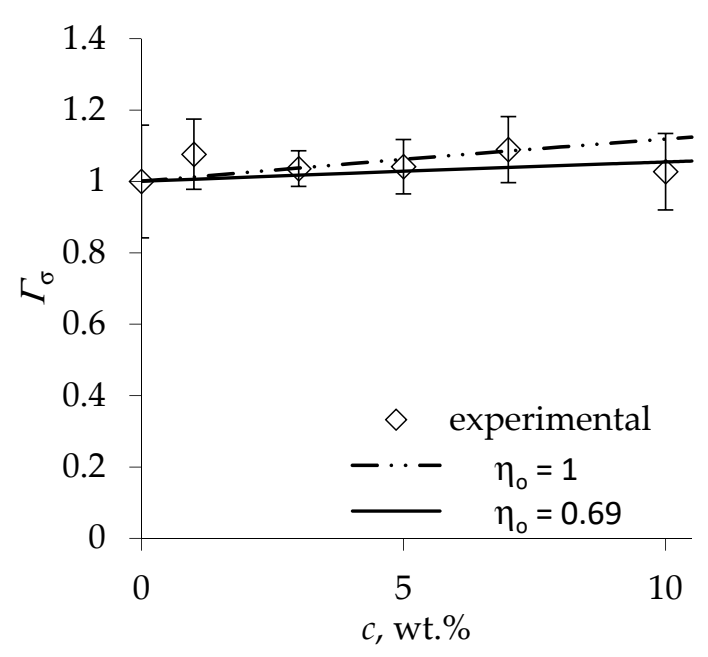

(b)

Figure 3. Mechanical reinforcement efficiency factors of composite foam (a) stiffness and (b) strength versus MCC fiber loading.

To quantitatively evaluate the accuracy of prediction of the mechanical reinforcement efficiency factor for stiffness, the relative root mean square (RMS) error is calculated as follows:

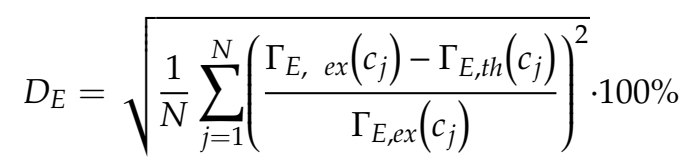

where $\Gamma_{E, e x}\left(c_{j}\right)$ denotes the stiffness reinforcement efficiency factor determined from foam test data at a filler loading $c_{j}$ by Equation (5), $\Gamma_{E, t h}\left(c_{j}\right)$ is the respective predicted value according to Equation (4), and $N$ is the number of filler loading levels considered. For strength, the relative RMS error $D_{\sigma}$ is calculated in the same way upon the substitution of $\Gamma_{E}$ by $\Gamma_{\sigma}$ in Equation (20). The results are presented in Table 3. It is seen that the relative RMS error is less than 5\% for the MCC fiber-filled foams.

Table 3. Relative root mean square error of prediction of the mechanical reinforcement efficiency by cellulose micro- and nanofibers.

\begin{tabular}{cccc}
\hline \multirow{2}{*}{ Relative RMS Error } & \multicolumn{3}{c}{ Fibrous Cellulose Filler } \\
\cline { 2 - 4 } & MCC Fibers & Cellulose Whiskers [42] & Cellulose Nanofibrils [45] \\
\hline$D_{\mathrm{E}}, \%$ & 4.9 & 10.9 & 18.4 \\
$D_{\sigma}, \%$ & 3.8 & 5.9 & 34.1 \\
\hline
\end{tabular}

Considerably higher gains in the mechanical properties of rigid low-density PU foams have been reported when applying cellulose whiskers as the filler, see e.g., [42,46]. Sucrose- and glycerol-based polyols and polymeric diphenylmethane diisocyanate (MDI) were used to produce the foams [42]. Composite foams were obtained by applying cellulose whiskers, derived from softwood craft pulp by sulfuric acid hydrolysis, as a filler at loadings $c=0.25 \mathrm{wt} \%, 0.5 \mathrm{wt} \%, 0.75 \mathrm{wt} \%$, and $1 \mathrm{wt} \%$ of the total weight of polyols and MDI. Considering the tensile properties, the highest gains were obtained at $1 \mathrm{wt} \%$ loading of nanocellulose and amounted to about $227 \%$ for modulus and $99 \%$ for strength [42]. Notably, foam density also increased by ca. $52 \%$, from $53.2 \mathrm{~kg} / \mathrm{m}^{3}$ for neat foams to $82 \mathrm{~kg} / \mathrm{m}^{3}$ for foams with the highest whisker content.

Since not only foam strength and stiffness but also density was markedly affected by the nanofiller, the density effect needs to be taken into account when evaluating reinforcement efficiency. In the absence 
of experimental data on density dependence of the neat foam stiffness and strength, we employed density exponent values $n_{E}=2$ and $n_{\sigma}=1.5$ derived using rectangular unit cell model [28]. Since the presence of whiskers apparently affected cell size while no effect on the shape anisotropy of cells was reported in [42], $R_{c f}=R$ was assumed. The mechanical reinforcement efficiency factors evaluated by Equations (5) and (9) are presented in Figure 4. It is seen that the maximum gain in nanocomposite foam stiffness, when corrected for density variation, becomes ca. $40 \%$, and for strength $-14 \%$.

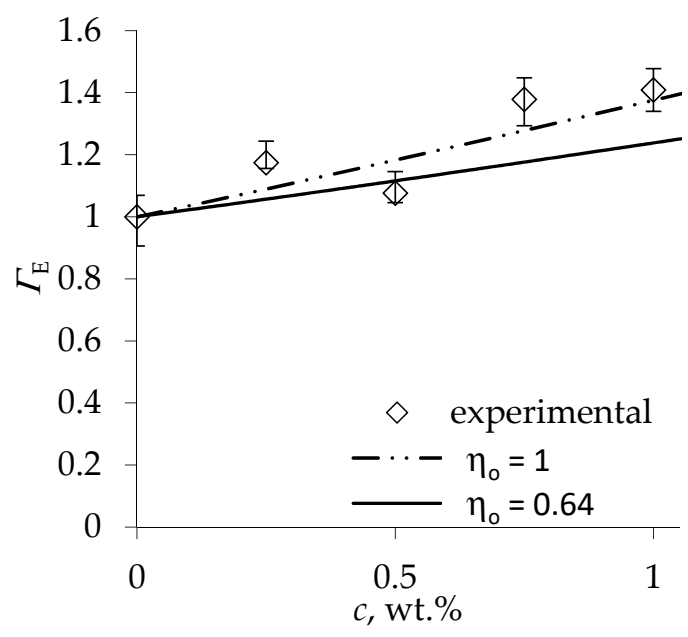

(a)

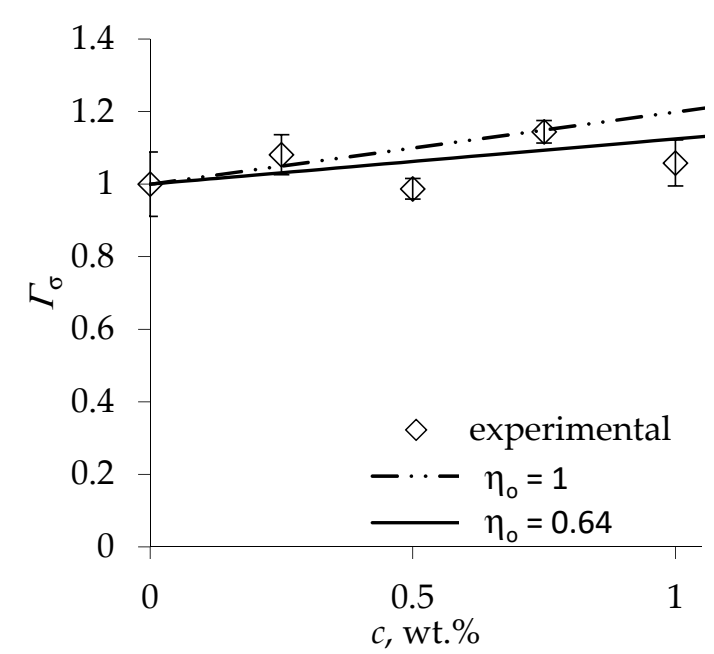

(b)

Figure 4. Mechanical reinforcement efficiency factors of composite foam (a) stiffness and (b) strength versus cellulose whisker [42] loading.

For evaluation of the theoretical reinforcement efficiency factors of the nanocomposite, we employed the following estimates of filler properties: longitudinal whisker modulus $E_{A}=151 \mathrm{GPa}$ and strength $\sigma_{A}=7.5 \mathrm{GPa}$ [10], shear modulus $G_{A}=15.5 \mathrm{GPa}$ [47], and the aspect ratio of whiskers, produced by sulfuric acid hydrolysis of softwood craft pulp, $\kappa=50$ [48]. The monolithic PU properties were assumed to be the same as quoted above. Since the total weight of polyols and MDI comprised $96 \mathrm{wt} \%$ of the neat PU foam formulation excluding the physical blowing agent [42], the volume fraction of nanocellulose in the PU polymer was expressed as $v_{f}=c /\left(c+\varrho_{c e l} / 0.96 \varrho_{s}\right)$ where the nanocellulose density $\varrho_{c e l}=1600 \mathrm{~kg} / \mathrm{m}^{3}$. Nanocomposite foams had slightly larger density than the MCC-filled ones; hence, the orientation factor calculated using the average expansion ratio of foams was smaller, amounting to $\eta_{o}=0.64$. The predicted efficiency factors $\Gamma_{E}$ and $\Gamma_{\sigma}$ of nanocomposite foams according to Equations (4) and (8) for $\eta_{0}=0.64$ are plotted in Figure 4 by solid lines. The modest increase in the reinforcement efficiency for strength with nanocellulose loading predicted via the strut strength model agrees very well with the $\Gamma_{\sigma}$ values derived from foam tests, as seen in Figure $4 \mathrm{~b}$, as also indicated by the respective relative RMS error of prediction shown in Table 3. However, the mechanical reinforcement efficiency of foam stiffness is closer to that predicted for fully aligned whiskers $\left(\eta_{o}=1\right)$.

Considerably higher loadings of cellulose nanofibrils (CNF), $20 \mathrm{wt} \%$ and $30 \mathrm{wt} \%$ of the combined polyol and CNF weight, were applied for filling rigid low-density PU foams in [45]. Thus, the weight fraction of CNF in foams, according to the formulation presented in [45], amounted to $10.3 \mathrm{wt} \%$ and $15.4 \mathrm{wt} \%$. A reduction in the cell size of composite foams but no effect on cell shape anisotropy was reported [45]; therefore, $R_{c f}=R$ was assumed when estimating the reinforcement efficiency factors by Equations (5) and (9). Substantial gains in the foam stiffness and strength in tension, bending, and compression were achieved, as shown in Figure 5. The variability of stiffness reinforcement efficiency among different loading modes seen in Figure $5 \mathrm{a}$ is likely to reflect the inter-batch variability of foams, the rate of growth of the bending and compressive stiffness with CNF loading being similar. However, the specific tensile stiffness at the highest filler loading was reported to increase by a factor 
of 10 [45], which appears to be inconsistent with the rest of data; hence, the respective data point was excluded from further analysis as an outlier. The reinforcement efficiency for strength, Figure $5 b$, exhibits marked scatter, tensile strength of foams benefiting from filler the least and compressive strength - the most. Such an effect is apparently caused by differing sensitivity in tension, bending, and compression to the stress concentrations caused by filler agglomerates in cell struts, defects in foam morphology, and superficial flaws introduced during the cutting of specimens.

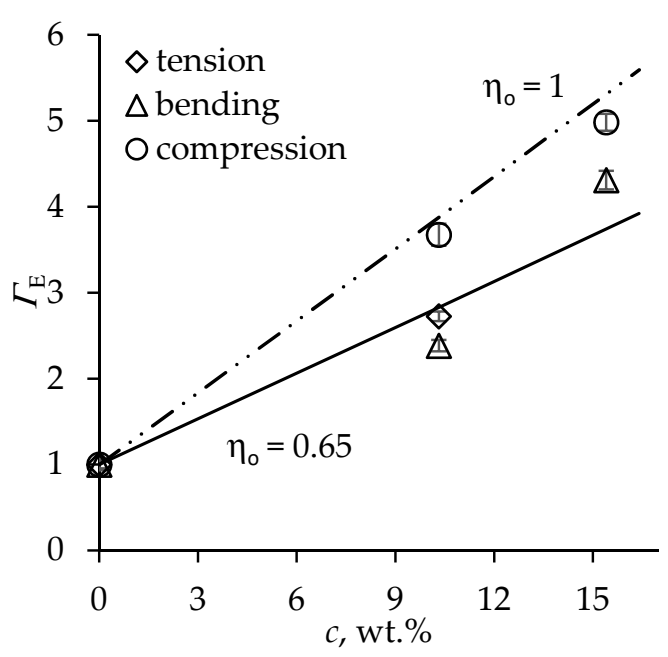

(a)

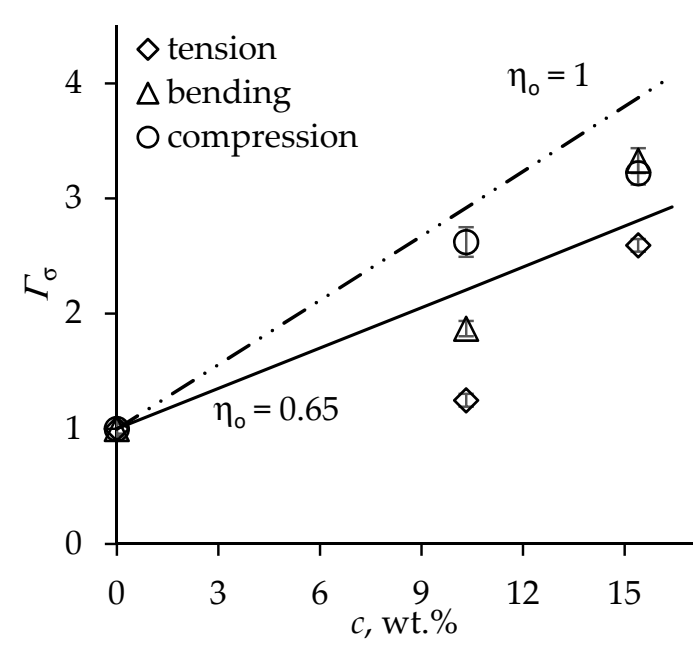

(b)

Figure 5. Mechanical reinforcement efficiency factors of composite foam (a) stiffness and (b) strength versus cellulose nanofibril [45] loading.

For theoretical estimation of the reinforcement efficiency factors of CNF-filled foams, we employed the longitudinal modulus $E_{A}=88 \mathrm{GPa}$ determined for hardwood-derived CNF [49] and strength $\sigma_{A}=1.6 \mathrm{GPa}$ [50]. Due to the large aspect ratio of CNFs [10], the fiber length efficiency factor was taken as $\eta_{o E}=\eta_{l \sigma}=1$. The volume fraction of CNF in the PU polymer was expressed via the weight fraction as $v_{f}=c /\left(c+(1-c) \varrho_{c e l} / \varrho_{s}\right)$. The properties of monolithic PU were assumed to be the same as above. The theoretical mechanical reinforcement efficiency of foams as a function of CNF loading according to Equations (4) and (8) is plotted in Figure 5 by solid lines for the partial alignment of fibers in the cell struts $\left(\eta_{o}=0.65\right)$. It is seen that the predicted dependence of reinforcement efficiency on filler loading reasonably closely reflects the experimental trend, although the relative RMS error values are considerably larger, see Table 3, which is mainly due to the scatter among results for different loading modes. The predicted reinforcement efficiency for $\eta_{0}=1$, shown by broken lines in Figure 5, demonstrates the $\Gamma_{E}, \Gamma_{\sigma}$ values theoretically attainable at a perfect CNF alignment.

Clearly, a much higher mechanical reinforcement efficiency of rigid PU foams can be achieved by the comparatively stiff, strong, and high aspect ratio cellulose whiskers and nanofibrils than by MCC fibers, even when corrected for variation in foam density and morphology, and for the selection of appropriate reinforcing filler for a given foam application, functionality, cost, and sustainability issues have to be balanced. The reinforcement efficiency factors for foam stiffness and strength can be applied as a tool in filler selection that reveals the intrinsic mechanical reinforcement effect of the filler.

\section{Conclusions}

MCC fibers have been considered as filler for enhancing the tensile stiffness and strength of rigid low-density bio-based PU foams, varying MCC loading up to $10 \mathrm{wt} \%$. Foam stiffness increase by ca. $20 \%$ and strength - by $9 \%$ has been achieved. Theoretical estimates of the mechanical reinforcement efficiency factors for foam stiffness and strength are derived based on the rule-of-mixtures type of relations for the mechanical properties of struts of filled foams, taking into account the orientation of 
anisometric filler particles along the longitudinal direction of foam struts during foaming. The approach presented has been applied to the analysis of mechanical properties of low-density rigid PU foams reinforced by MCC fibers as well as by cellulose whiskers and nanofibrils. It is shown that reasonably close estimates of the mechanical reinforcement efficiency can be obtained by the proposed model.

Author Contributions: Conceptualization, J.A. and U.C.; methodology, M.K. and U.C.; formal analysis, J.A.; investigation, M.K.; writing-original draft preparation, J.A.; writing-review and editing, M.K. and U.C.; project administration, U.C. All authors have read and agreed to the published version of the manuscript.

Funding: The modeling activities were funded by ERA-NET Cofund M-ERA.Net 2 project "Development of Biobased Cryogenic Insulation Modified with Nanocrystalline Cellulose" (Bio4Cryo), while the experimental work was supported by the European Regional Development Fund via project 2010/0290/2DP/2.1.1.1.0/10/ APIA/VIAA/053 “Development of Rigid Polyisocyanurate Biofoams Filled with Natural Materials".

Conflicts of Interest: The authors declare no conflict of interest. The funders had no role in the design of the study; in the collection, analyses, or interpretation of data; in the writing of the manuscript, or in the decision to publish the results.

\section{References}

1. Gama, N.V.; Ferreira, A.; Barros-Timmons, A. Polyurethane Foams: Past, Present, and Future. Materials 2018, 11, 1841. [CrossRef] [PubMed]

2. Chiacchiarelli, L. Sustainable, nanostructured, and bio-based polyurethanes for energy-efficient sandwich structures applied to the construction industry. In Biomass, Biopolymer-Based Materials, and Bioenergy; Elsevier BV: Amsterdam, The Netherlands, 2019; pp. 135-160.

3. Joshi, M.; Adak, B.; Butola, B.S. Polyurethane nanocomposite based gas barrier films, membranes and coatings: A review on synthesis, characterization and potential applications. Prog. Mater. Sci. 2018, 97, 230-282. [CrossRef]

4. Stirna, U.; Beverte, I.; Yakushin, V.; Cabulis, U. Mechanical properties of rigid polyurethane foams at room and cryogenic temperatures. J. Cell. Plast. 2011, 47, 337-355. [CrossRef]

5. Saha, M.; Kabir, E.; Jeelani, S. Enhancement in thermal and mechanical properties of polyurethane foam infused with nanoparticles. Mater. Sci. Eng. A 2008, 479, 213-222. [CrossRef]

6. Cabulis, U.; Sevastyanova, I.; Andersons, J.; Beverte, I. Rapeseed oil-based rigid polyisocyanurate foams modified with nanoparticles of various type. Polimery 2014, 59, 207-212. [CrossRef]

7. Dwan'Isa, J.-P.L.; Mohanty, A.; Misra, M.; Drzal, L.T.; Kazemizadeh, M. Biobased polyurethane and its composite with glass fiber. J. Mater. Sci. 2004, 39, 2081-2087. [CrossRef]

8. Kim, S.H.; Park, H.C.; Jeong, H.M.; Kim, B.K. Glass fiber reinforced rigid polyurethane foams. J. Mater. Sci. 2010, 45, 2675-2680. [CrossRef]

9. Yakushin, V.; Stirna, U.; Bel'Kova, L.; Deme, L.; Sevastyanova, I. Properties of rigid polyurethane foams filled with milled carbon fibers. Mech. Compos. Mater. 2011, 46, 679-688. [CrossRef]

10. Moon, R.J.; Martini, A.; Nairn, J.; Simonsen, J.; Youngblood, J.P. Cellulose nanomaterials review: Structure, properties and nanocomposites. Chem. Soc. Rev. 2011, 40, 3941. [CrossRef]

11. Berglund, L.A.; Peijs, T. Cellulose Biocomposites-From Bulk Moldings to Nanostructured Systems. MRS Bull. 2010, 35, 201-207. [CrossRef]

12. Siró, I.; Plackett, D. Microfibrillated cellulose and new nanocomposite materials: A review. Cellulose 2010, 17, 459-494. [CrossRef]

13. Turk, J.; Oven, P.; Poljanšek, I.; Lešek, A.; Knez, F.; Rebec, K.M. Evaluation of an environmental profile comparison for nanocellulose production and supply chain by applying different life cycle assessment methods. J. Clean. Prod. 2020, 247, 119107. [CrossRef]

14. Arvidsson, R.; Nguyen, D.; Svanström, M. Life Cycle Assessment of Cellulose Nanofibrils Production by Mechanical Treatment and Two Different Pretreatment Processes. Environ. Sci. Technol. 2015, 49, 6881-6890. [CrossRef] [PubMed]

15. Hussain, S.; Kortschot, M. Polyurethane foam mechanical reinforcement by low-aspect ratio micro-crystalline cellulose and glass fibres. J. Cell. Plast. 2014, 51, 59-73. [CrossRef] 
16. Mosiewicki, M.A.; Rojek, P.; Michałowski, S.; Aranguren, M.I.; Prociak, A. Rapeseed oil-based polyurethane foams modified with glycerol and cellulose micro/nanocrystals. J. Appl. Polym. Sci. 2014, 132, 41602. [CrossRef]

17. Kurańska, M.; Prociak, A.; Michalowski, S.; Cabulis, U.; Kirpluks, M. Microcellulose as a natural filler in polyurethane foams based on the biopolyol from rapeseed oil. Polimery 2016, 61, 625-632. [CrossRef]

18. Shen, J.; Han, X.; Lee, L.J. Nanoscaled Reinforcement of Polystyrene Foams using Carbon Nanofibers. J. Cell. Plast. 2006, 42, 105-126. [CrossRef]

19. Okamoto, M.; Nam, P.H.; Maiti, P.; Kotaka, T.; Nakayama, T.; Takada, M.; Ohshima, M.; Usuki, A.; Hasegawa, N.; Okamoto, H. Biaxial Flow-Induced Alignment of Silicate Layers in Polypropylene/Clay Nanocomposite Foam. Nano Lett. 2001, 1, 503-505. [CrossRef]

20. Nam, P.H.; Maiti, P.; Okamoto, M.; Kotaka, T.; Nakayama, T.; Takada, M.; Ohshima, M.; Usuki, A.; Hasegawa, N.; Okamoto, H. Foam processing and cellular structure of polypropylene/clay nanocomposites. Polym. Eng. Sci. 2002, 42, 1907-1918. [CrossRef]

21. Strauss, W.; D'Souza, N. Supercritical CO2 Processed Polystyrene Nanocomposite Foams. J. Cell. Plast. 2004, 40, 229-241. [CrossRef]

22. Shaayegan, V.; Ameli, A.; Wang, S.; Park, C.B. Experimental observation and modeling of fiber rotation and translation during foam injection molding of polymer composites. Compos. Part A Appl. Sci. Manuf. 2016, 88, 67-74. [CrossRef]

23. Wang, S.; Ameli, A.; Shaayegan, V.; Kazemi, Y.; Huang, Y.; Naguib, H.E.; Park, C.B. Modelling of Rod-Like Fillers' Rotation and Translation near Two Growing Cells in Conductive Polymer Composite Foam Processing. Polymers 2018, 10, 261. [CrossRef] [PubMed]

24. Gimenez, R.B.; Leonardi, L.; Cerrutti, P.; Amalvy, J.; Chiacchiarelli, L.M. Improved specific thermomechanical properties of polyurethane nanocomposite foams based on castor oil and bacterial nanocellulose. J. Appl. Polym. Sci. 2017, 134, 44982. [CrossRef]

25. Septevani, A.A.; Evans, D.A.C.; Hosseinmardi, A.; Martin, D.J.; Simonsen, J.; Conley, J.F.; Annamalai, P.K. Atomic Layer Deposition of Metal Oxide on Nanocellulose for Enabling Microscopic Characterization of Polymer Nanocomposites. Small 2018, 14, 1803439. [CrossRef] [PubMed]

26. Shishkina, O.; Lomov, S.V.; Verpoest, I.; Gorbatikh, L. Modeling of elastic properties of cell-wall material in nanoclay-reinforced foams. J. Cell. Plast. 2015, 52, 107-130. [CrossRef]

27. Andersons, J.; Kirpluks, M.; Cabulis, U. Modelling the Strength of Cellulose Nanofiber-Filled Rigid Low-Density PU Foams. Key Eng. Mater. 2019, 827, 159-164. [CrossRef]

28. Gibson, L.J.; Ashby, M.F. Cellular Solids: Structure and Properties; Cambridge University Press: Cambridge, UK, 1997.

29. Lobos, J.; Velankar, S.S. How much do nanoparticle fillers improve the modulus and strength of polymer foams? J. Cell. Plast. 2014, 52, 57-88. [CrossRef]

30. Siegmann, A.; Kenig, S.; Alperstein, D.; Narkis, M. Mechanical behavior of reinforced polyurethane foams. Polym. Compos. 1983, 4, 113-119. [CrossRef]

31. Hamilton, A.R.; Thomsen, O.T.; Madaleno, L.A.; Jensen, L.R.; Rauhe, J.C.M.; Pyrz, R. Evaluation of the anisotropic mechanical properties of reinforced polyurethane foams. Compos. Sci. Technol. 2013, 87, 210-217. [CrossRef]

32. Reitz, D.; Schuetz, M.; Glicksman, L. A Basic Study of Aging of Foam Insulation. J. Cell. Plast. 1984, 20, 104-113. [CrossRef]

33. Ahern, A.; Verbist, G.; Weaire, D.; Phelan, R.; Fleurent, H. The conductivity of foams: A generalisation of the electrical to the thermal case. Colloids Surf. A Physicochem. Eng. Asp. 2005, 263, 275-279. [CrossRef]

34. Andersons, J.; Kirpluks, M.; Stiebra, L.; Cabulis, U. Anisotropy of the stiffness and strength of rigid low-density closed-cell polyisocyanurate foams. Mater. Des. 2016, 92, 836-845. [CrossRef]

35. Nairn, J.A. Generalized Shear-Lag Analysis Including Imperfect Interfaces. Adv. Compos. Lett. 2004, 13, 263-274. [CrossRef]

36. Hine, P.; Parveen, B.; Brands, D.; Caton-Rose, F. Validation of the modified rule of mixtures using a combination of fibre orientation and fibre length measurements. Compos. Part A Appl. Sci. Manuf. 2014, 64, 70-78. [CrossRef]

37. Fukuda, H.; Chou, T.-W. A probabilistic theory of the strength of short-fibre composites with variable fibre length and orientation. J. Mater. Sci. 1982, 17, 1003-1011. [CrossRef] 
38. Andersons, J.; Spārniňš, E.; Joffe, R. Stiffness and strength of flax fiber/polymer matrix composites. Polym. Compos. 2006, 27, 221-229. [CrossRef]

39. Dafalias, Y.F. Orientation distribution function in non-affine rotations. J. Mech. Phys. Solids 2001, 49, 2493-2516. [CrossRef]

40. Eichhorn, S.J.; Young, R.J. The Young's modulus of a microcrystalline cellulose. Cellulose 2001, 8, $197-207$. [CrossRef]

41. Roberts, R.J.; Rowe, R.C.; York, P. The Poisson's ratio of microcrystalline cellulose. Int. J. Pharm. 1994, 105, 177-180. [CrossRef]

42. Li, Y.; Ren, H.; Ragauskas, A.J. Rigid polyurethane foam/cellulose whisker nanocomposites: Preparation, characterization, and properties. J. Nanosci. Nanotechnol. 2011, 11, 6904-6911. [CrossRef]

43. Luo, X.; Mohanty, A.; Misra, M. Water-Blown Rigid Biofoams from Soy-Based Biopolyurethane and Microcrystalline Cellulose. J. Am. Oil Chem. Soc. 2012, 89, 2057-2065. [CrossRef]

44. Andersons, J.; Filipova, I.; Kirpluks, M.; Cabulis, U. Evaluation of the Apparent Interfacial Shear Strength of Nanocellulose/PVA Composites. Key Eng. Mater. 2018, 774, 54-59. [CrossRef]

45. Leng, W.; Pan, B. Thermal Insulating and Mechanical Properties of Cellulose Nanofibrils Modified Polyurethane Foam Composite as Structural Insulated Material. Forests 2019, 10, 200. [CrossRef]

46. Li, Y.; Ragauskas, A.J. Ethanol organosolv lignin-based rigid polyurethane foam reinforced with cellulose nanowhiskers. RSC Adv. 2012, 2, 3347. [CrossRef]

47. Chen, P.; Ogawa, Y.; Nishiyama, Y.; Ismail, A.E.; Mazeau, K. Linear, non-linear and plastic bending deformation of cellulose nanocrystals. Phys. Chem. Chem. Phys. 2016, 18, 19880-19887. [CrossRef] [PubMed]

48. Araki, J.; Wada, M.; Kuga, S.; Okano, T. Flow properties of microcrystalline cellulose suspension prepared by acid treatment of native cellulose. Colloids Surf. A Physicochem. Eng. Asp. 1998, 142, 75-82. [CrossRef]

49. Zhai, L.; Kim, H.C.; Kim, J.W.; Kang, J.; Kim, J. Elastic moduli of cellulose nanofibers isolated from various cellulose resources by using aqueous counter collision. Cellulose 2018, 25, 4261-4268. [CrossRef]

50. Saito, T.; Kuramae, R.; Wohlert, J.; Berglund, L.A.; Isogai, A. An Ultrastrong Nanofibrillar Biomaterial: The Strength of Single Cellulose Nanofibrils Revealed via Sonication-Induced Fragmentation. Biomacromolecules 2012, 14, 248-253. [CrossRef] 